\title{
螺旋状下降的复式断面明渠中 紊动结构与次生流动的数值模拟。
}

\author{
邵学军 王 虹 陈 智
}

(清华大学水利系, 北京 100084)

摘要采用正交曲线坐标下建立的紊流控制方程, 对弯道水流中由离心力和 紊动应力联合驱动的二次流结构建立了数学模型. 将螺旋下降的明渠流动条件 下的数值模拟结果与如下试验资料进行了对比: ( i ) 交错边滩式矩形断面弯道明 渠流动, (ii)复式断面顺直明渠流动. 计算中采用了 3 种不同的 Reynolds 应力计算 方法, 包括 Launder 和 Ying(LY)及 Naot 和 Rodi(NR)的代数应力模型及运用非线 性 $k-\varepsilon$ 模型计算紊动黏性系数的 $\mathrm{SY}$ 模型, 在各种弯道曲率和边界条件下, 对不 同紊流模型的二次流结构数值模拟结果精度进行了评估. 研究表明, LY 和 SY 模 型数值模拟计算得到的二次流结构和紊动应力分布与试验数据均能达到趋势上 的符合, 能够模拟出紊动应力和弯道流动中的离心力对二次流结构形成的影响.

\section{关键词 紊流模型 弯道流动 复式断面 正交曲线坐标}

复式断面顺直明渠中滩、槽水流的时均与紊动特性及其相互间的作用，已有 不少试验研究 ${ }^{[1 ~ 5]}$, 获得了较完整的流速分布和紊动应力实测资料. 对复式断面 弯道流动结构的试验研究还很有限. Keily 采用一维激光流速仪量测了弯道紊流, 由于量测手段的限制没有直接得到紊动应力和次生环流结构 ${ }^{1)}$. Shiono 和 Muto 采用二维激光流速仪在具有顺直堤坝的弯曲河道上的复式断面中量测了紊动应 力和次生流动结构 ${ }^{[6]}$. 已有的数值模拟多以简单断面(如正方形)弯道流动或复式 断面顺直明渠为对象 (如文献[7]和[8]), 在对不同曲率的弯道或不同形式的复式 断面情况下的紊动结构和次生流动模拟方面还缺少深入的研究.

本文采用正交曲线坐标下的三维 Reynolds 控制方程和常用非线性 $k-\varepsilon$ 模型及

2003-06-10 收稿, 2003-10-14 收修改稿

*国家自然科学基金资助项目(批准号: 50179015, 59879009, 50221903)

1) Kiely G K. An experimental study of overbank flow in straight and meandering compound channels. PhD Thesis, University College, Cork, Ireland, 1989 
代数应力模型, 模拟呈螺旋状下降的明渠中不同曲率、不同复式断面形状时的紊 动结构, 如图 1(a). 该种明渠流动中, 边壁的弯曲特性、比降沿程不变, 断面上的 紊动结构和次生流动具有沿程均匀的特性. 而图 1(b)所示天然冲积河流中交错边 滩形成的弯道中, 紊动的结构和螺旋流方向沿程有周期性的变化. 螺旋状下降的 明渠弯道可以看作是对天然交错边滩弯道的一种概化或简化. 由于技术条件的 限制, 尚不能实际建造一条螺旋状下降的弯道明渠进行试验验证, 但可将前者的 模拟结果与后者的实测结果进行比较, 以定性判别数值模拟的可靠性.

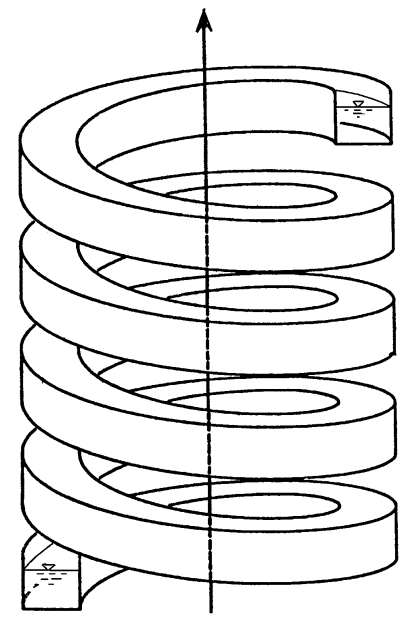

(a)

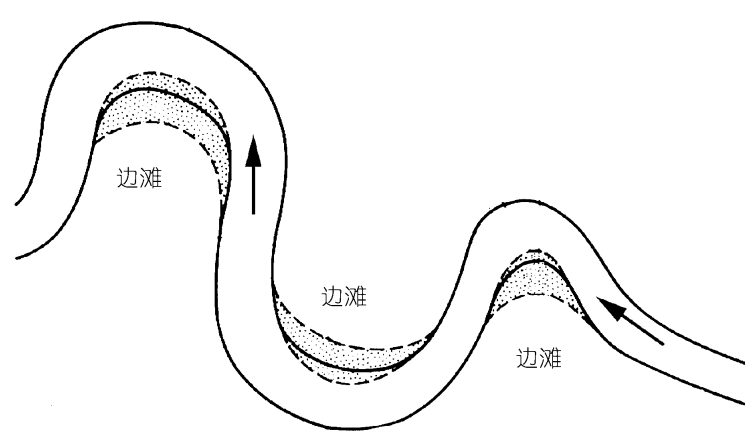

(b)

图 1 螺旋状下降式明渠弯道与天然冲积河流中的交错边滩式弯道

(a) 呈螺旋状无穷下降的弯道明渠, (b) 天然冲积河流中的交错边滩式弯道

\section{1 控制方程}

计算中采用的不同弯道曲率用弯曲系数 $\alpha=R_{\mathrm{L}} / B$ 来表示, 其中, $R_{\mathrm{L}}$ 是弯道左 边壁的弯曲半径, $B$ 是弯曲段的宽度, 如图 2. 定义滩地宽度系数 $\beta=b / B$ 和相对水 深 $h_{\mathrm{r}}=h / H$, 其中 $b$ 是滩地宽度, $H$ 是主槽水深, $h$ 是滩地水深, $h_{1}$ 是滩槽差, 如 图 2(a). 在图 3 所示的坐标系中, $\xi$ 和 $\eta$ 是水平面上的正交曲线坐标轴, $z$ 是垂直 坐标轴, 向上为正. 在 $\xi$ 和 $\eta$ 方向上的微小增量可分别记为 $\mathrm{d} s_{1}$ 和 $\mathrm{d} s_{2}$, 并表达为如 下形式:

$$
\mathrm{d} s_{1}=h_{1}(\xi, \eta) \delta \xi, \mathrm{d} s_{2}=h_{2}(\xi, \eta) \delta \eta .
$$

函数 $f(\xi, \eta, z)$ 的导数可写为

$$
\frac{\partial f}{\partial s_{1}}=\frac{1}{h_{1}} \frac{\partial f}{\partial \xi}, \frac{\partial f}{\partial s_{2}}=\frac{1}{h_{2}} \frac{\partial f}{\partial \eta}, \frac{\partial f}{\partial z}=\frac{\partial f}{\partial z} .
$$

因此可将控制方程写为如下形式:

连续方程 


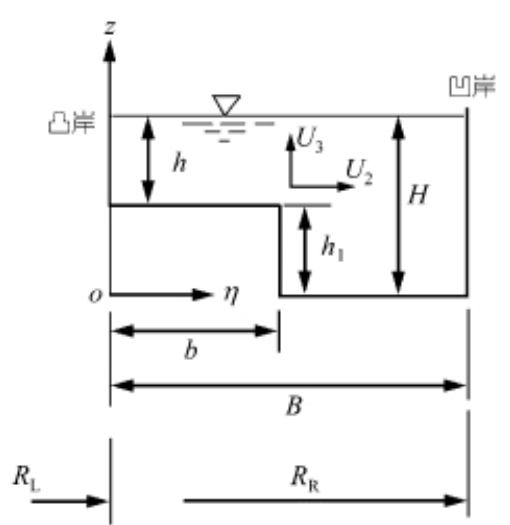

(a)

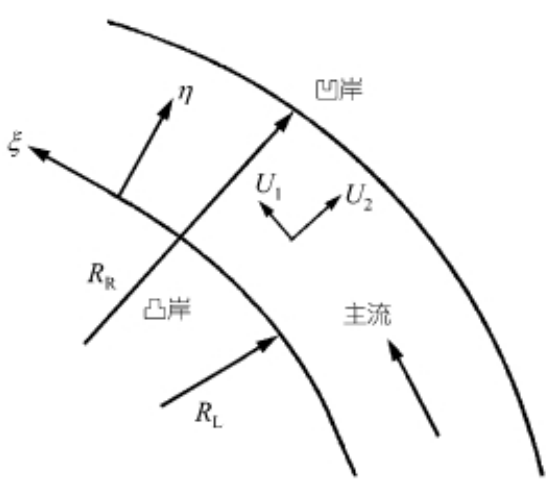

(b)

图 2 复式断面弯道的形态

(a) 弯道的断面形态, (b) 弯道的平面形态

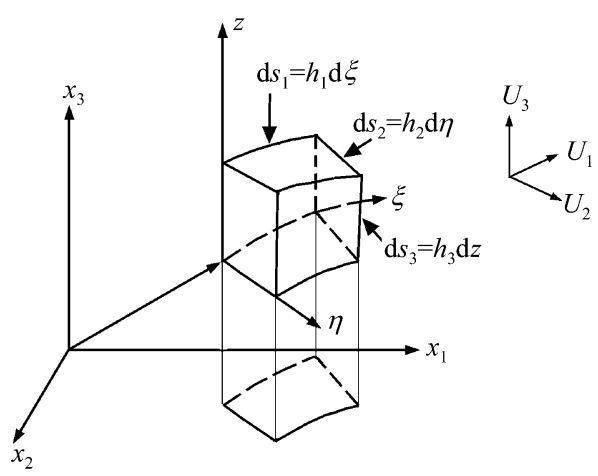

图 3 曲线坐标的定义

$$
\frac{\partial U_{1}}{\partial s_{1}}+\frac{\partial U_{2}}{\partial s_{2}}+\frac{\partial U_{3}}{\partial z}+\frac{U_{1}}{r_{\eta}}+\frac{U_{2}}{r_{\xi}}=0
$$

运动方程

$$
\begin{aligned}
& U_{1} \frac{\partial U_{1}}{\partial s_{1}}+U_{2} \frac{\partial U_{1}}{\partial s_{2}}+U_{3} \frac{\partial U_{1}}{\partial z}+\frac{U_{1} U_{2}}{r_{\xi}}-\frac{U_{2}^{2}}{r_{\eta}} \\
= & -\frac{\partial}{\partial s_{1}}\left(\frac{p}{\rho}+W\right)-\frac{\partial \overline{u_{1} u_{1}}}{\partial s_{1}}-\frac{\partial \overline{u_{1} u_{2}}}{\partial s_{2}}-\frac{\partial \overline{u_{1} u_{3}}}{\partial z}-\frac{2 \overline{u_{1} u_{2}}}{r_{\xi}}-\frac{\overline{u_{1} u_{1}}-\overline{u_{2} u_{2}}}{r_{\eta}} \\
& U_{1} \frac{\partial U_{2}}{\partial s_{1}}+U_{2} \frac{\partial U_{2}}{\partial s_{2}}+U_{3} \frac{\partial U_{2}}{\partial z}+\frac{U_{1} U_{2}}{r_{\eta}}-\frac{U_{1}^{2}}{r_{\xi}} \\
= & -\frac{\partial}{\partial s_{2}}\left(\frac{p}{\rho}+W\right)-\frac{\partial \overline{u_{1} u_{2}}}{\partial s_{1}}-\frac{\partial \overline{u_{2} u_{2}}}{\partial s_{2}}-\frac{\partial \overline{u_{2} u_{3}}}{\partial z}-\frac{2 \overline{u_{1} u_{2}}}{r_{\eta}}-\frac{\overline{u_{2} u_{2}}-\overline{u_{1} u_{1}}}{r_{\xi}},
\end{aligned}
$$




$$
\begin{aligned}
& U_{1} \frac{\partial U_{3}}{\partial s_{1}}+U_{2} \frac{\partial U_{3}}{\partial s_{2}}+U_{3} \frac{\partial U_{3}}{\partial z} \\
= & -\frac{\partial}{\partial z}\left(\frac{p}{\rho}+W\right)-\frac{\partial \overline{u_{1} u_{3}}}{\partial s_{1}}-\frac{\partial \overline{u_{1} u_{3}}}{\partial s_{2}}-\frac{\partial \overline{u_{3} u_{3}}}{\partial z}-\frac{\overline{u_{1} u_{3}}}{r_{\eta}}-\frac{\overline{u_{2} u_{3}}}{r_{\xi}}
\end{aligned}
$$

其中,

$$
\frac{1}{r_{\xi}}=\frac{1}{h_{1} h_{2}} \frac{\partial h_{1}}{\partial \eta}, \frac{1}{r_{\eta}}=\frac{1}{h_{1} h_{2}} \frac{\partial h_{2}}{\partial \xi},
$$

式中, $U_{1}, U_{2}, U_{3}$ 和 $u_{1}, u_{2}, u_{3}$ 分别是正交曲线坐标 $\xi$ (纵向)、 $\eta$ (横向)和 $z$ (垂向)方 向上时均流速和脉动流速; $h_{1}, h_{2}$ 和 $h_{3}$ 为 Lame 系数; $p$ 为时均压强; $W$ 为重力势; $\rho$ 为流体密度.

方程(3) (5)中出现的 Reynolds 应力 $\overline{u_{2}^{2}}, \overline{u_{3}^{2}}, \overline{u_{1} u_{2}}, \overline{u_{1} u_{3}}$ 和 $\overline{u_{2} u_{3}}$ 是影响次生 流动形态的因子, 其中, $\overline{u_{3}^{2}}-\overline{u_{2}^{2}}$ 和 $\overline{u_{2} u_{3}}$ 是主导因子 ${ }^{[9]}$. 因此, 在数值模拟中必须 准确地计算出 $\overline{u_{3}^{2}}-\overline{u_{2}^{2}}$ 和 $\overline{u_{2} u_{3}}$, 才能够使计算结果合乎实际情况. 本项研究中对 方程中的 Reynolds 应力表达式分别采用 3 种不同模型, 其在直角坐标下的形式如 下所述.

\subsection{LY 代数应力模型}

Launder 和 $\mathrm{Ying}^{[10]}$ 对紊动应力项 $\overline{u_{3}^{2}}-\overline{u_{2}^{2}}$ 和 $\overline{u_{2} u_{3}}$ 单独推导了计算式, 而对主 流方向上的应力项采用标准的浴黏性模型计算, 次生流动方向上的应力通过求 解紊动动能 $k$ 的输运方程来计算:

$$
\begin{aligned}
& \overline{u_{3}^{2}}-\overline{u_{2}^{2}}=-c l^{2}\left[\left(\frac{\partial U_{1}}{\partial x_{3}}\right)^{2}-\left(\frac{\partial U_{1}}{\partial x_{2}}\right)^{2}\right], \\
& \overline{u_{2} u_{3}}=-c l^{2}\left(\frac{\partial U_{1}}{\partial x_{3}}\right)\left(\frac{\partial U_{1}}{\partial x_{2}}\right), \\
& \overline{u_{1} u_{3}}=-v_{\mathrm{T}} \frac{\partial U_{1}}{\partial x_{3}}, \overline{u_{1} u_{2}}=-v_{\mathrm{T}} \frac{\partial U_{1}}{\partial x_{2}}, \\
& v_{\mathrm{T}}=c_{v} k^{1 / 2} l,
\end{aligned}
$$

其中, $l$ 是紊动长度比尺, $c$ 和 $c_{v}$ 是无量纲常数, 一般分别取为 0.01 和 0.22 .

\section{$1.2 N R$ 代数应力模型 ${ }^{[11]}$}

该模型通过附加涡黏性项与表面附近修正, 考虑了次生流动速度的梯度对 紊动应力的影响:

$$
\overline{u_{3}^{2}}=\frac{k}{c_{1}+2 c_{3} f_{2}}\left[\frac{2}{3}\left(a-\frac{1}{2} b+c_{1}-1\right)+\frac{b}{\varepsilon}\left(\overline{u_{1} u_{3}} \frac{\partial U_{1}}{\partial x_{3}}-\overline{u_{1} u_{2}} \frac{\partial U_{1}}{\partial x_{2}}\right)\right]-2 v_{t} \frac{\partial U_{3}}{\partial x_{3}},
$$




$$
\begin{gathered}
\overline{u_{2}^{2}}=\frac{k}{c_{1}}\left[\frac{2}{3}\left(a-\frac{1}{2} b+c_{1}-1\right)+c_{3} f_{2} \frac{\overline{u_{3}^{2}}}{k}+\frac{b}{\varepsilon}\left(\overline{u_{1} u_{2}} \frac{\partial U_{1}}{\partial x_{2}}-\overline{u_{1} u_{3}} \frac{\partial U_{1}}{\partial x_{3}}\right)\right]-2 v_{t} \frac{\partial U_{2}}{\partial x_{2}} \\
\overline{u_{2} u_{3}}=\frac{b}{\left(c_{1}+\frac{3}{2} c_{3} f_{2}\right)} \frac{k}{\varepsilon}\left(\overline{u_{1} u_{2}} \frac{\partial U_{1}}{\partial x_{3}}+\overline{u_{1} u_{3}} \frac{\partial U_{1}}{\partial x_{2}}\right)-v_{t}\left(\frac{\partial U_{3}}{\partial x_{2}}+\frac{\partial U_{2}}{\partial x_{3}}\right) \\
v_{t}=c_{\mu} \frac{k^{2}}{\varepsilon} \\
c_{\mu}=0.09
\end{gathered}
$$

其中, $a=0.7637-0.06 f_{1}, b=0.1091+0.06 f_{1}, c_{1}=1.5-0.5 f_{1}, c_{3}=0.10 f_{2}, f_{1}$ 和 $f_{2}$ 是距水面无量纲距离的函数.

\section{$1.3 \mathrm{SY}$ 代数应力模型}

该模型又称为非线形 $k-\varepsilon$ 模型 ${ }^{[12]}$. 沿主流方向的应力表达式与 LY 模型相同, 次生流动方向上的表达式有所不同:

$$
\begin{gathered}
\tau_{i j}=-\frac{2}{3} k \delta_{i j}+\rho k^{1 / 2} l D_{i j}+C_{D} \rho l^{2}\left(D_{i m} D_{m j}-\frac{1}{3} D_{m n} D_{m n} \delta_{i j}\right) \\
+C_{E} \rho l^{2}\left(\dot{D}_{i j}-\frac{1}{3} \dot{D}_{m m} \delta_{i j}\right), \\
D_{i j}=\frac{1}{2}\left(\frac{\partial U_{i}}{\partial x_{j}}+\frac{\partial U_{j}}{\partial x_{i}}\right)(i, j=1,2,3), \\
\dot{D}_{i j}=\frac{\partial D_{i j}}{\partial t}+U \cdot \nabla D_{i j}-\frac{\partial U_{i}}{\partial x_{k}} D_{k j}-\frac{\partial U_{j}}{\partial x_{k}} D_{k i}(k=1,2,3),
\end{gathered}
$$

其中, $C_{D}$ 和 $C_{E}$ 是无量纲常数, 均为 $1.68, l$ 是紊动长度比尺, 可由下式计算:

$$
l=c \frac{k^{3 / 2}}{\varepsilon} .
$$

通过坐标转换, 可将上述模型的应力表达式转换为曲线坐标形式 ${ }^{1)}$.

\section{2 求解方法及流动条件}

本文中以 SIMPLER 方法对上述模型进行数值求解, 使用交错网格, 离散方 法为: 源项采用二阶中心差格式, 对流项和扩散项采用幂函数格式. 由于假设流 动沿程是均匀恒定的, 忽略所有沿流向的导数项. 求解时从假定的流速场开始, 将断面二维网格中的解沿流向推进求解. 利用连续方程和动量方程求解流速分 量和压力分量后, 解出 $k$-方程和 $\varepsilon$-方程, 并由前述代数表达式计算 Reynolds 应力. 达到稳定的流场时求解过程结束. 边界条件采用 Launder 和 Spalding 的壁面函数

1) 陈 智. 明渠弯道复式断面流动中二次流的数值模拟方法研究. 清华大学硕士学位论文, 2000 年 5 月 
法处理平行于壁面的流速 (垂直于壁面的流速为零 $)^{[13]}$. 自由水面处的独立变量除 $\varepsilon$ 外均采用对称条件.

\section{3 矩形断面弯道流动的计算结果}

首先利用上述模型对螺旋状无穷下降的矩形断面弯道中的充分发展紊流进 行了计算. 计算中采用的参数为：断面宽 $B=0.3 \mathrm{~m}$, 水深 $H=0.2 \mathrm{~m}$; 水力坡降 $J$ $=1 / 1500$; 弯曲系数 $R_{\mathrm{L}} / B=1.5,3.5,5.0$. 断面网格点布置为 $30 \times 20$ 个. 由于螺旋 状无穷下降的矩形断面弯道目前尚不能在试验中实现, 本文中用于比较的试验 数据是 $\mathrm{Chang}^{1)}$ 在弯曲系数为 $R_{\mathrm{L}} / B=3.1$ 的矩形断面交错弯道中的试验结果, 其试 验采用几个 $90^{\circ}$ 弯道方向交错地联结在一起以模拟天然河流中的交错边滩式弯 道, 断面平均流速为 $0.366 \mathrm{~m} / \mathrm{s}$, 试验水深为 $0.115 \mathrm{~m}$. 为实现二维流动状态, 采用 的水槽宽为 $2.34 \mathrm{~m}$, 水槽中心线的弯曲半径为 $8.53 \mathrm{~m}$.

\section{1 时均流速的计算结果}

图 4 和 5 所示为数值计算结果与矩形断面交错弯道中实测的无量纲时均流速 之间的比较, 其中 $U_{0}$ 是断面平均流速. 由图 4 可见, 3 种不同的紊流模型给出的

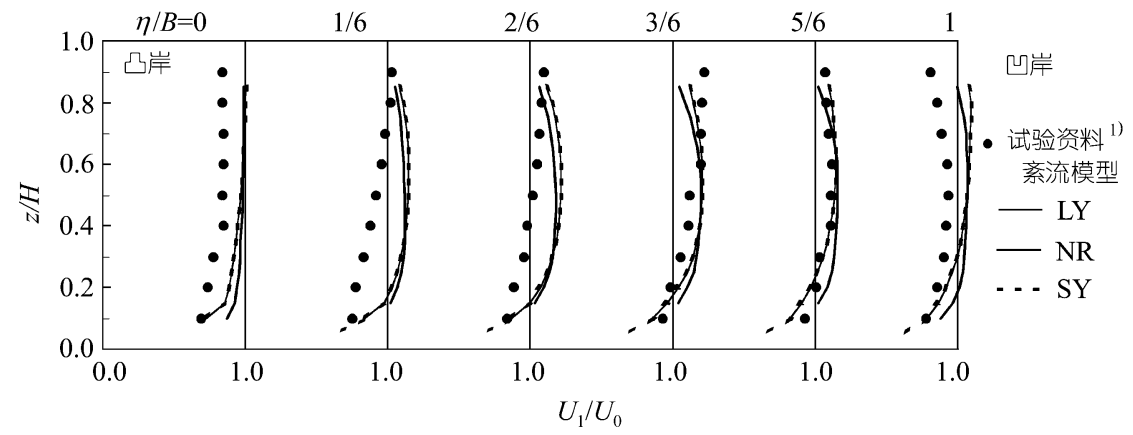

图 4 数值计算结果与实测的纵向时均流速比较 $\left(U_{1} / U_{0}\right)$

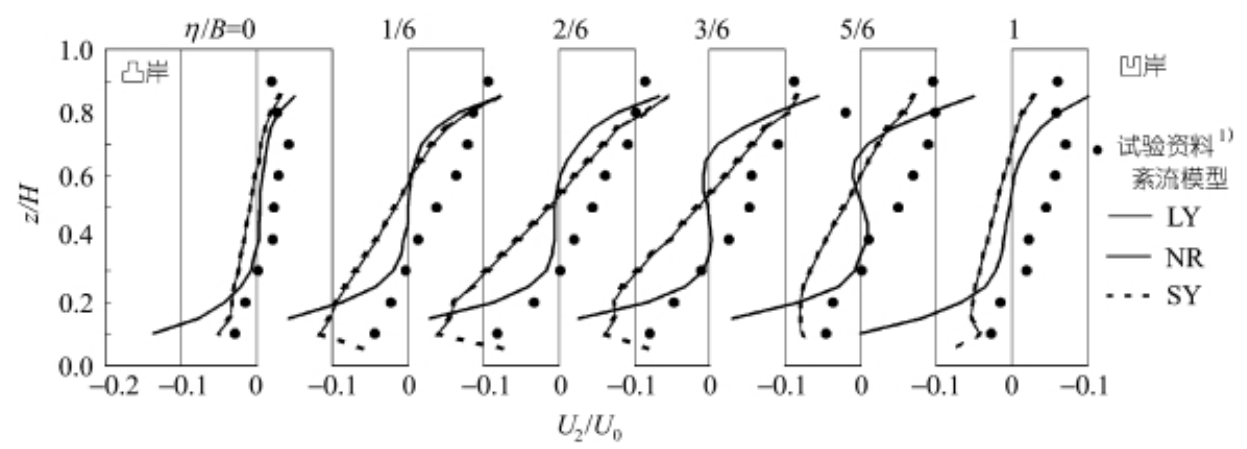

图 5 数值计算结果与实测的横向时均流速比较 $\left(U_{2} / U_{0}\right)$

1) Chang Y C. Lateral mixing in meandering channels. PhD thesis, Univ of Iowa, Iowa City, Iowa, 1971 
纵向流速结果均比较接近. 试验条件下的紊动结构在空间上变化较大, 而计算条 件下的紊动结构沿程是均匀的、横向上变化也较缓. 计算结果中少数垂线与实测 资料符合较好，而其余垂线上有明显误差.

由图 5 所示的横向流速计算结果可见, 采用 LY 和 SY 模型计算得到的横向 流速沿垂线的梯度与实测值相近, 但二次环流的中心位置高于实测值, 其偏差尚 认为与各自的水流条件有关. 而采用 NR 模型计算得到的二次环流中心范围过大, 即使考虑到计算条件与试验条件的差异，从定性趋势上看横向流速梯度也不符 合实测结果.

图 6 所示为采用不同弯曲系数和不同紊流模型时二次流场的计算结果, 计算
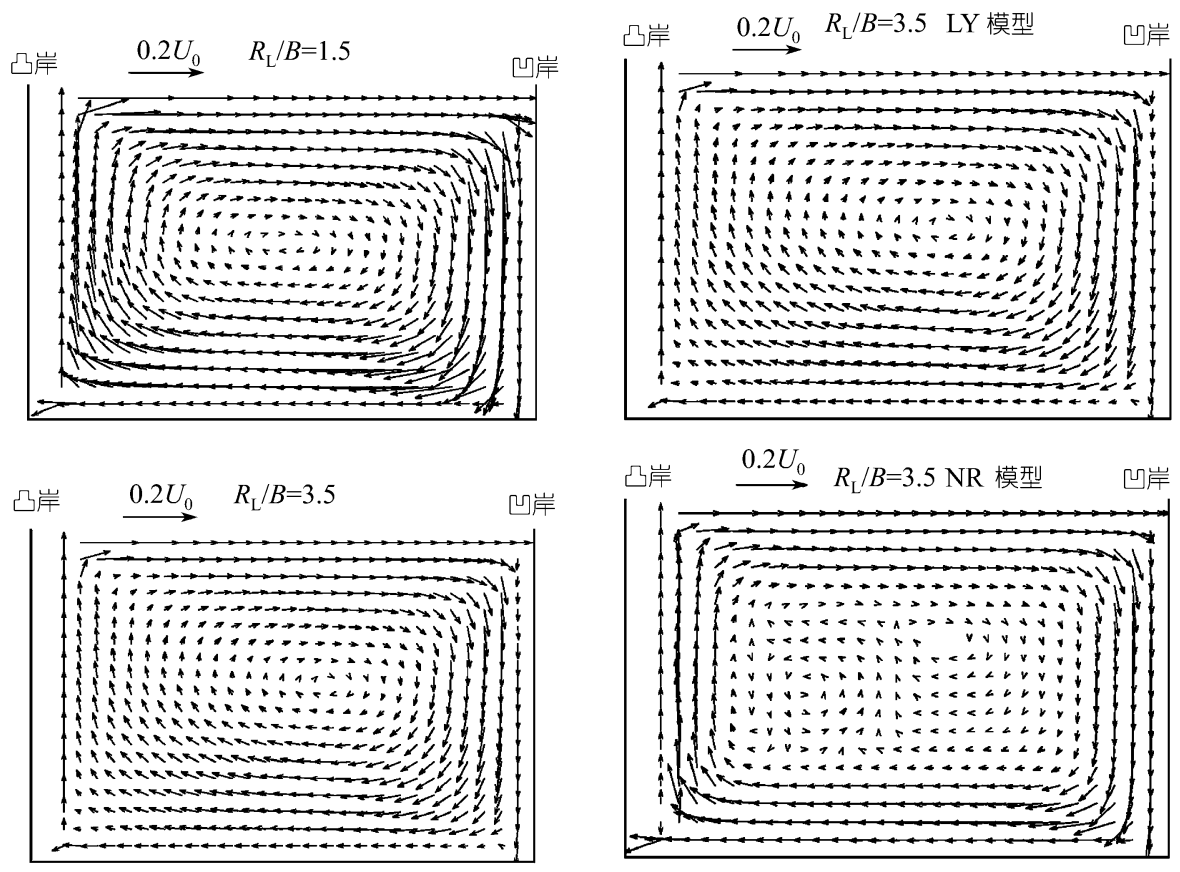

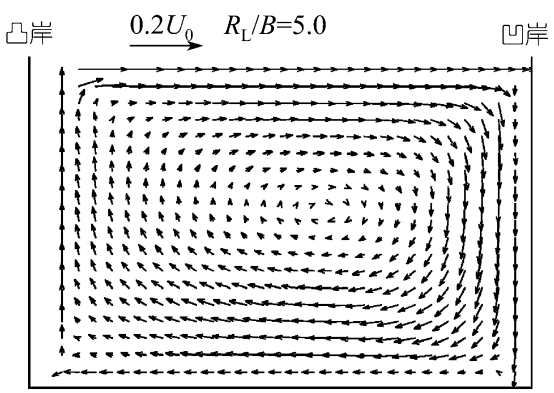

(a)

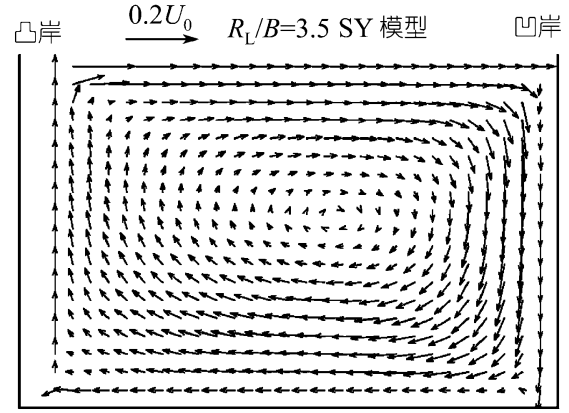

(b)

图 6 采用不同弯曲系数和不同紊流模型时二次流场的计算结果

(a) 不同弯曲系数下 LY 紊流模型的二次流场计算结果; (b) 弯曲系数 $R_{\mathrm{L}} / B=3.5$ 时采用不同紊流模型的 二次流场计算结果 
得到的均为单一环流. 图 6(a) 是采用 $\mathrm{LY}$ 模型计算的 3 种不同弯曲系数 $\left(R_{\mathrm{L}} / B=1.5\right.$, $3.5,5.0)$ 情况下的二次流场, 图 6(b) 是弯曲系数 $R_{\mathrm{L}} / B=3.5$ 时采用 3 种不同紊流模 型得到的二次流场. 由图 $6(\mathrm{a})$ 可见, 弯曲系数 $R_{\mathrm{L}} / B$ 越小, 二次流流速越大, 二次 流动越强烈. 图 6(b)则表明不同紊流模型对二次流的计算结果存在明显差异. LY 模型和非线性 $k-\varepsilon$ 模型计算得出的二次流动环流中心点偏高, 但横向流速沿垂线 的梯度与实测结果比较接近, 即二次流速的量值从边界附近向环流中心是逐渐 减小的. NR 模型的横向流速和流速梯度计算结果与实测资料的差别较大, 表现 在横向流速计算值在边界附近大于测量结果, 在自由表面和边界附近有一个较 高的流速带. 随后向环流中心的方向急剧减小, 出现一个较大的低流速区, 并有 几个不同方向的小环流出现. 横向流速沿垂线的梯度有较大变化(横向流速在垂 线上不是均匀的减小).

\section{2 紊动特征值}

由于弯道二次流动的部分成因是紊动应力驱动, 因此紊动应力的计算精度 决定了二次流场的计算是否准确. 图 7 所示是 $\mathrm{Chang}^{1}$ 在矩形断面交错弯道中实 测的横向流速脉动值与 3 种紊流模型计算结果的比较. 可见 3 个紊流模型的计算 结果彼此较为接近. 由于各自流动条件的差异, 计算值与实测值除在固壁边界处 比较符合外, 在核心流区中部和下部有明显偏离.
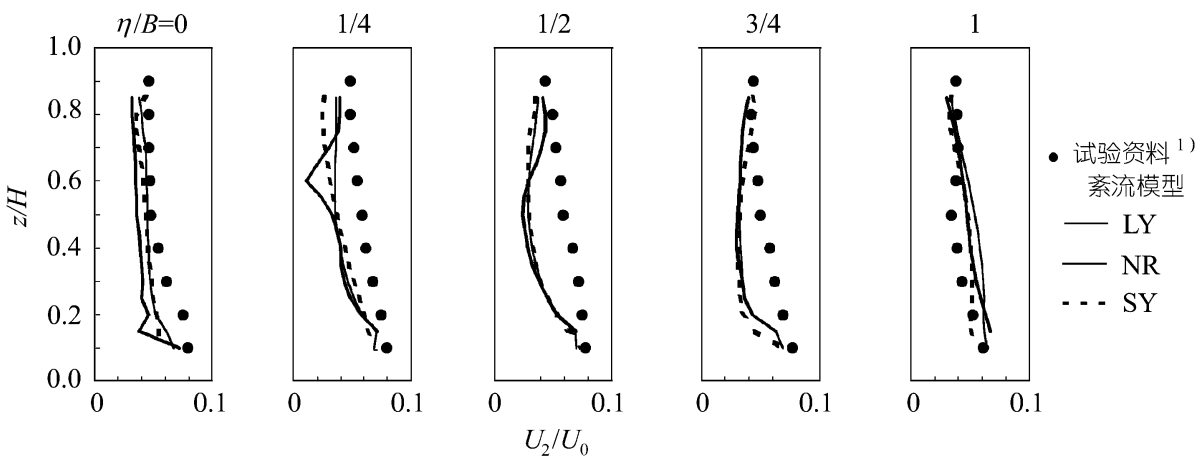

图 7 矩形断面弯道中 3 种紊流模型计算结果与实测横向流速脉动值 $U_{2} / U_{0}$ 的比较

\section{4 复式断面弯道流动的计算结果验证}

复式断面弯道流动计算中采用了 3 种弯曲系数 $R_{\mathrm{L}} / B=1.5,3.5,5.0$, 对不同的 滩地宽度系数 $\beta=b / B$ 及相对水深 $h_{\mathrm{r}}=h / H$ 的组合进行计算. 计算中用到的各种断 面形式总结如表 1 .

1) 见 69 页脚注 
表 1 数值计算采用的弯道复式断面形式

\begin{tabular}{cccccc}
\hline 编号 & $A_{1}$ & $A_{2}$ & $A_{3}$ & $A_{4}$ & $A_{5}$ \\
\hline$\beta=b / B$ & $1 / 2$ & $1 / 3$ & $1 / 2$ & $2 / 3$ & 0 \\
$h_{\mathrm{r}}=h / H$ & $1 / 2$ & $3 / 4$ & $1 / 4$ & $3 / 4$ & 1 \\
\hline
\end{tabular}

\section{1 复式断面弯道流动的二次流结构}

由顺直复式断面明渠的试验结果可知, 在主槽和滩地上的浴对将引发一股 强烈的二次流动, 其方向为从滩槽交界点起倾斜向上至自由水面处, 如图 $8^{[4]}$. 对 于相对水深 $h / H=0.5$ 的断面形态, 合成速度 $\left(U_{2}^{2}+U_{3}^{2}\right)^{1 / 2}$ 的最大量值约为 $0.04 U_{1 \max }$, 其中 $U_{1 \max }$ 是最大纵向流速.

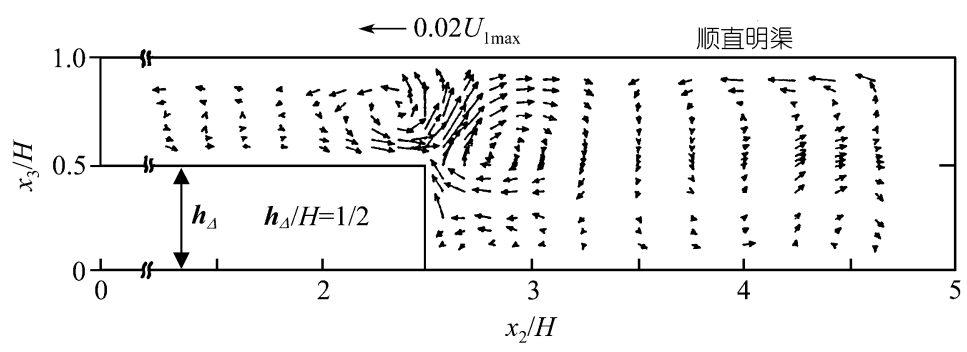

图 8 顺直复式断面明渠试验得到的主槽和滩地上浴对 ${ }^{[4]}$

图 9(a)和(b)所示是分别采用 SY 模型和 LY 模型计算 $A_{1}$ 断面得到的滩槽交界 处的二次流动, 计算条件为相对水深 $h / H=0.5$, 弯曲系数 $R_{\mathrm{L}} / B=5.0$. 可见, 弯道 计算结果接近于顺直渠道的实测流场, 计算得到的合成流速 $\left(U_{2}{ }^{2}+U_{3}{ }^{2}\right)^{1 / 2}$ 约为 $0.05 \sim 0.06 U_{1 \max }$, 大于顺直渠道的相对量值, 显然是由于弯道中离心力作用的影 响. SY 模型能够模拟出滩地上多个环流，与试验观测接近. 而 LY 模型计算结果 显示滩地上是单一环流, 但流动强度较大. 两种模型计算得到的主槽环流都是单 一的，由于离心力作用，环流为顺时针方向.
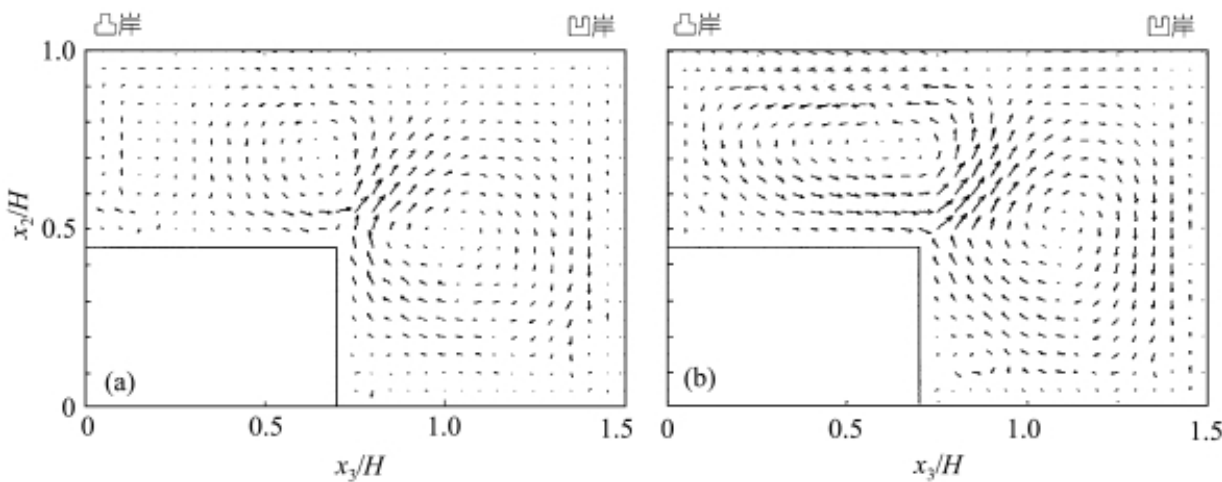

图 9 弯曲明渠滩槽交界处的二次流动 SY 模型(a)和 LY 模型(b)计算结果

图 10 所示相对滩高分别为 $h_{1} / H=0.25$ 和 $h_{1} / H=0.75$ 的试验结果表明, 在顺 直明渠中滩地的相对高度对二次流动的结构有较大影响 ${ }^{[4]}$. 相对滩高较小时, 滩 
地上有明显的环流存在, 而较大相对滩高(即滩上水深很小)的情况下, 滩地上不 易生成环流.

图 11 所示为采用 SY 模型对弯曲系数为 $R_{\mathrm{L}} / B=5.0$, 滩上相对水深分别为 $h / H$ $=0.75$ 和 0.25 的二次流动结构计算结果. 由图中可见, 两种不同的相对水深情况 下二次流结构均由一个主要环流控制, 这与顺直明渠试验的结果类似.
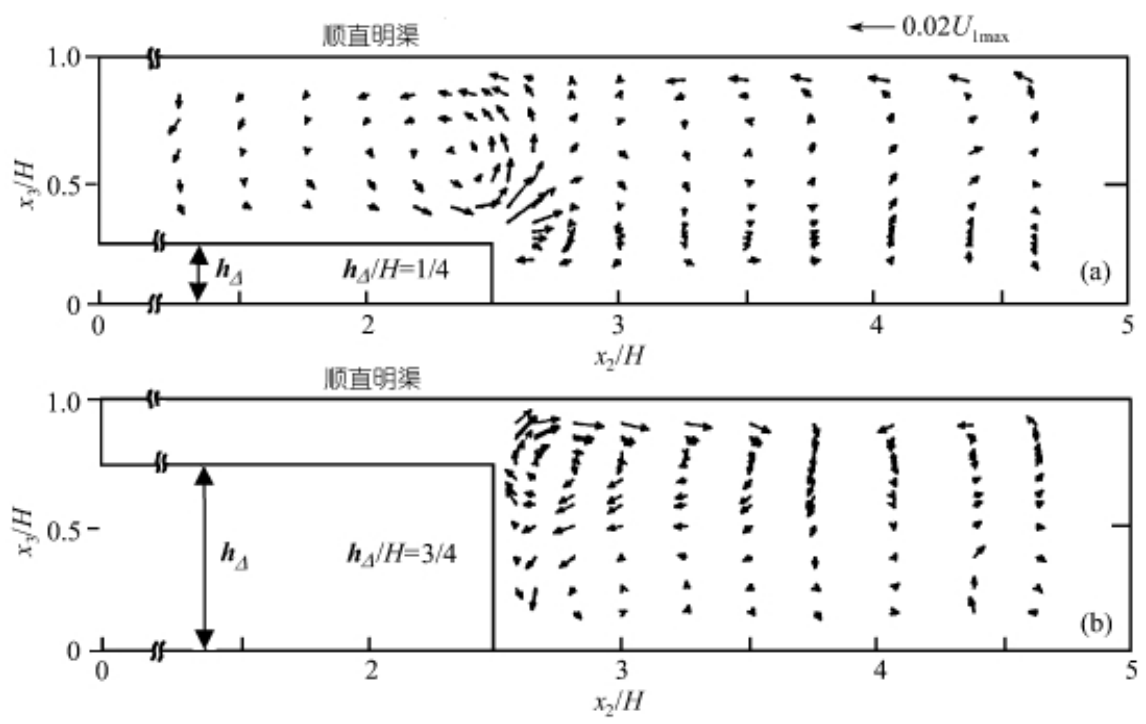

图 10 顺直明渠滩槽交界处的二次流动 SY 模型(a)和 LY 模型(b)计算结果

图 11 与顺直明渠测量结果(图 10)的不同之处是, 复式断面弯道流动中一旦 形成一个主要环流, 不论是在滩地上还是主槽中, 其环流方向都是顺时针的, 即 在自由水面处流动方向为从凸岸到凹岸, 而在底面上则相反. 可见, 弯道情况下 离心力的作用决定了主要环流(螺旋流)的方向, 在动床条件下会导致凹岸的后退 和凸岸边滩的形成. 次要的环流的方向则仍由紊动应力决定. 采用 NR 模型和 LY 模型的模拟计算也得到了类似结果.
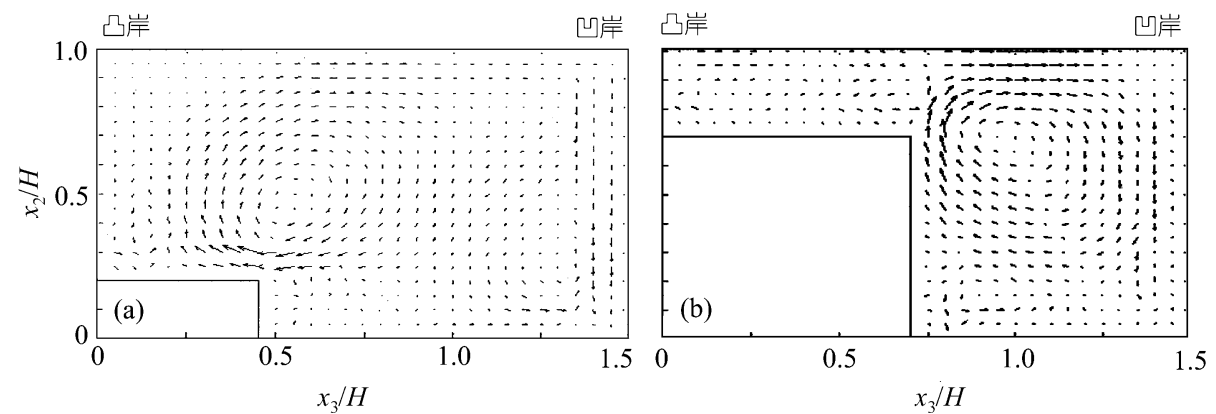

图 11 采用 SY 模型计算得到的不同相对水深下的二次流动结构

(a) $h / H=0.75$, (b) $h / H=0.25$ 


\section{2 复式断面弯道流动的主流流场}

图 12 所示为顺直明渠复式断面流动的实测主流等流速线 ${ }^{[4]}$. 由图中可以看 到，滩槽交界处二次流动把固壁附近的低速流体输向主流区，造成了一个明显的 低流速带, 将滩地流动和主槽流动分割开来. 图 13 所示为采用非线性 $k-\varepsilon(\mathrm{SY})$ 模 型和 LY 模型计算得到的主流等流速线, 其相对水深 $h / H=0.5$, 弯曲系数 $R_{\mathrm{L}} / B=$ 5.0 .

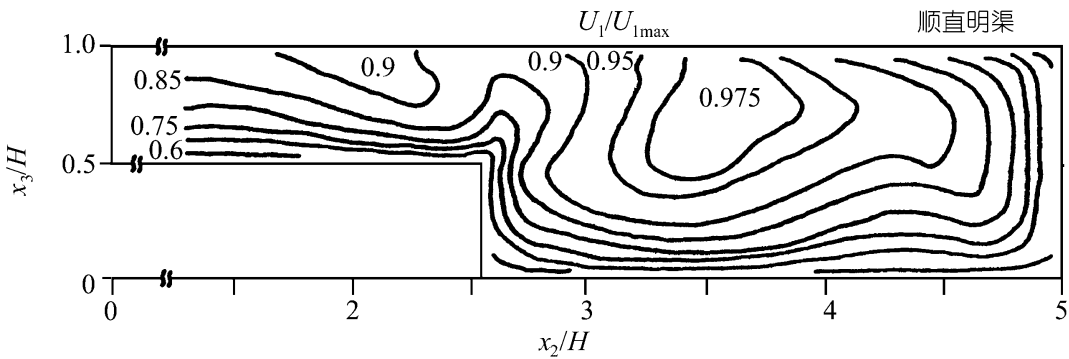

图 12 顺直明渠复式断面流动的实测主流等流速线分布 ${ }^{[4]}$

与图 12 相比, 靠近主槽凹岸一侧的流速梯度要高于滩地一侧，可以看出弯 道水流所受离心作用力的影响. LY 模型计算结果中低流速带的范围较大, 比较符 合实测情况，这实际上是由于该模型计算得到的滩槽交界处二次流强度较大，因 此动量传递较为明显. 而采用 SY 模型计算出的二次流强度较小, 动量传递弱, 因此所形成的低流速带范围稍小一些. 两种计算模型的计算结果中，弯道复式断 面流动最大主流流速均在自由表面处, 而顺直明渠的实测结果则表明最大主流 流速在自由水面以下. 这可能与计算中采用的某些人为限制条件有关(如刚盖假 设).
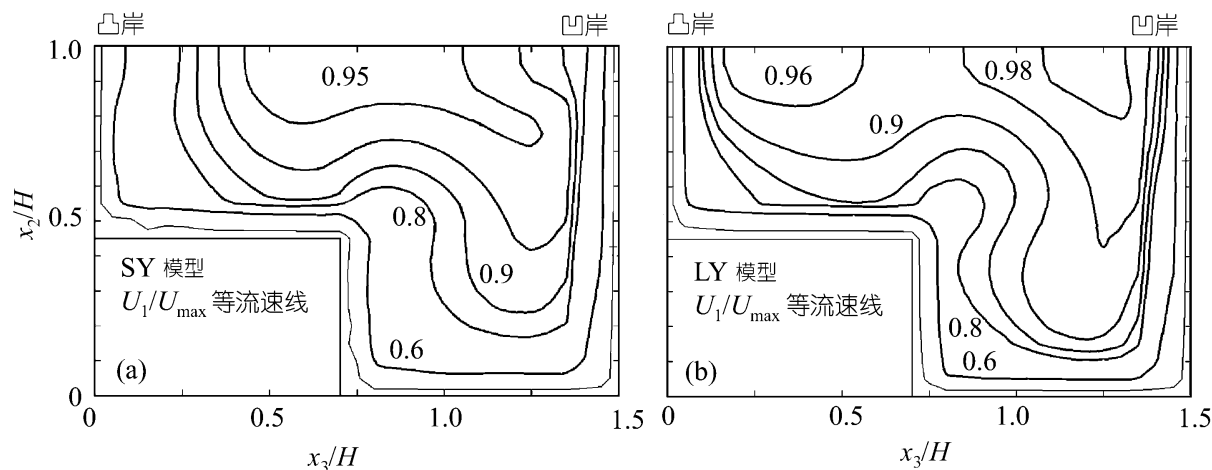

图 13 采用不同紊流模型得到的复式断面弯道流动主流等流速线分布

(a) 非线性 $k-\varepsilon$ (SY)模型, (b) LY 模型

\section{5 复式断面弯道流动的紊动特征值分布}

采用不同紊流模型，对复式断面弯道流动中垂向和横向紊动强度沿垂线的 
分布进行了计算, 并将其和顺直明渠情况的实测数据进行对比, 见图 14 和 15. 计 算条件为 $b / B=0.5, h / H=0.5$ 和 $R_{\mathrm{L}} / B=3.5$, 采用剪切流速 $U_{*}$ 进行无量纲化.
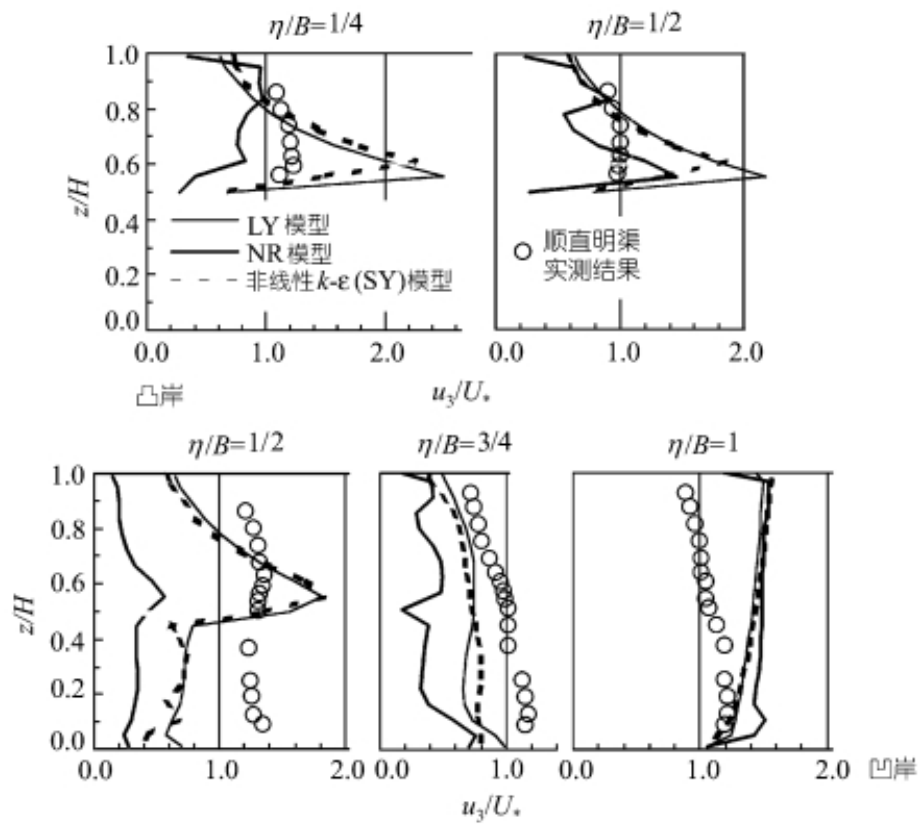

图 14 复式断面弯道流动中垂向紊动强度沿垂线分布的计算结果
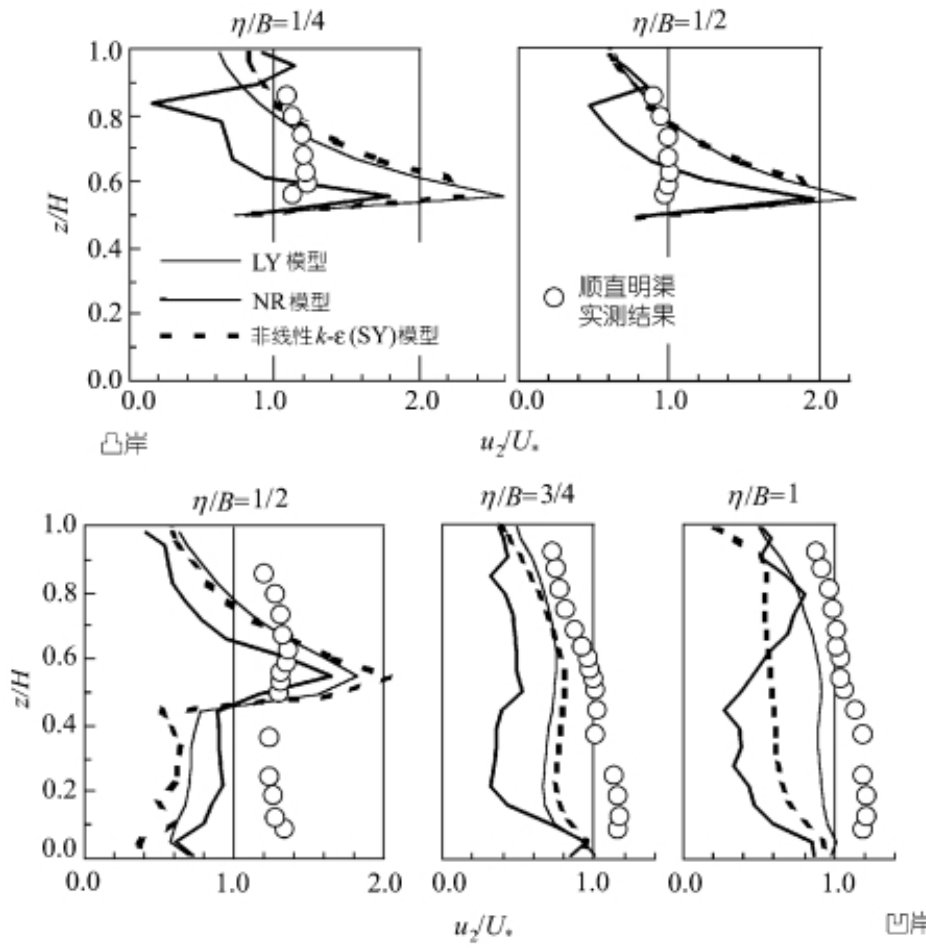

图 15 复式断面弯道流动中横向紊动强度沿垂线分布的计算结果 
由图 14 和 15 中可见, 弯道复式断面计算结果与顺直渠道量测结果的差别主 要表现在滩面和滩槽交界处附近. 在这些位置, 计算得出的弯道水流中的紊动强 度大大超过顺直渠道的量测结果, 而在矩形断面的情况下, 弯道计算及量测的紊 动强度结果与顺直渠道的情况差别并没有如此明显. 造成这种差别的原因可能 是弯道流动固有的特点, 但也不能排除紊流模型存在精度问题, 尚有待于进一步 研究.

实测的顺直渠道复式断面流动中 Reynolds 应力沿垂线的分布结果如图 16, 与计算得到的弯道复式断面流动中的结果(图 17)相比, 两者在复式断面的不同位 置上具有相同的分布趋势.

$x_{1} / B=0.1 / 0.4$

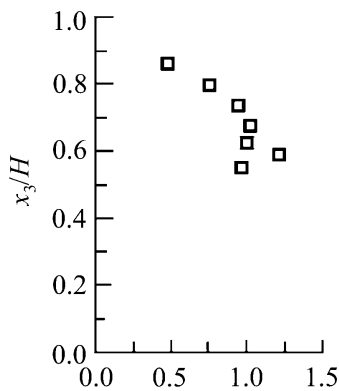

$0.245 / 0.4$

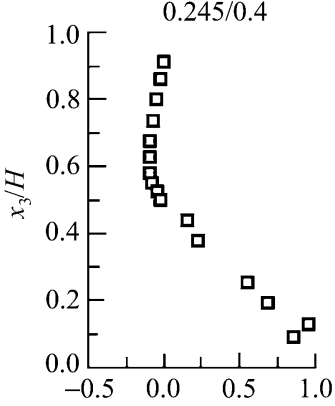

$0.195 / 0.4$

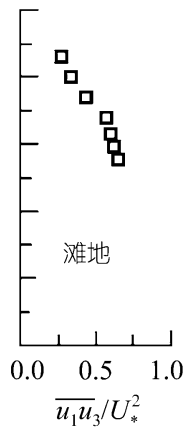

$0.3 / 0.4$
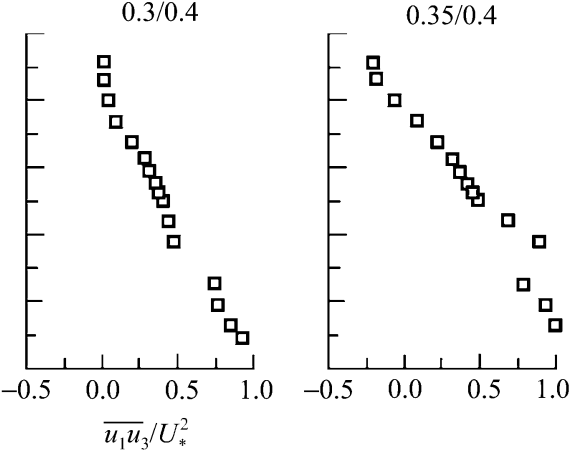

图 16 直道复式断面流动中 Reynolds 应力 $\overline{u_{1} u_{3}} / U_{*}^{2}$ 沿垂线分布的实测结果 ${ }^{[4]}$

\section{6 结语}

本文采用正交曲线坐标下的三维 Reynolds 控制方程, 结合非线性 $k-\varepsilon$ 模型或 代数应力模型, 在同时考虑紊动应力和弯道离心力作用的情况下, 对不同的曲率 和不同复式断面形状下，螺旋状下降的明渠弯道紊流的时均流动和紊动结构进 行了模拟. 由于技术条件的限制, 尚不能实际建造一条螺旋状下降的弯道明渠进 行试验验证, 本文只是将模拟结果与交错边滩型弯道的实测结果进行了对比, 定 性地判别数值模拟的可靠性. 最终可以得到如下结论:

( i ) 对弯道中的二次流动进行数值模拟时, 通过考虑离心力和紊动应力两 

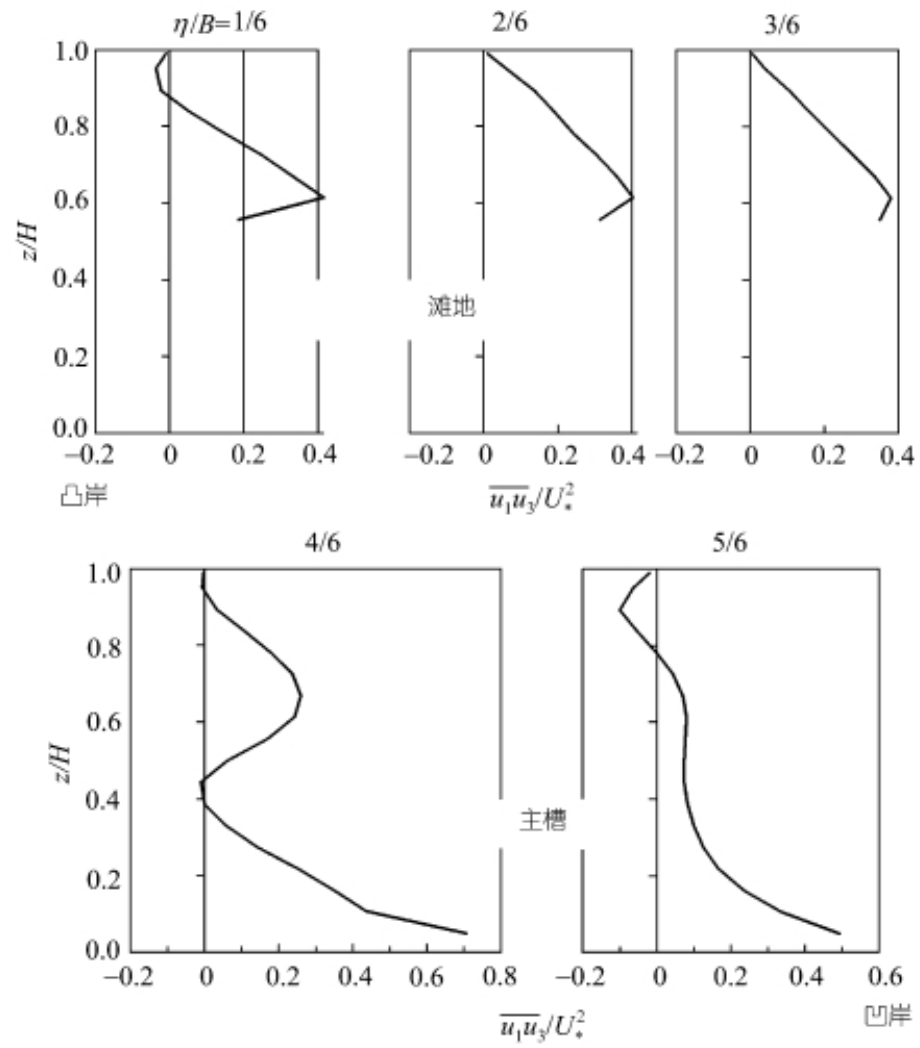

图 17 弯道复式断面流动中 Reynolds 应力 $\overline{u_{1} u_{3}} / U_{*}^{2}$ 沿垂线分布的计算结果 $\left(R_{\mathrm{L}} / B=3.5\right)$

者的共同影响, 可以反映出弯道曲率对环流强度的影响. 由于离心力的影响, 计 算得到的矩形断面弯道环流均为单一的.

(ii) 采用 3 种紊流模型计算得到的矩形断面弯道纵向时均流速和横向脉动 流速沿断面的分布差异不大, 且与交错边滩型弯道的实测数据比较接近. 在计算 横向时均流速时, LY 模型和 SY 模型给出的横向流速在垂线方向上的梯度与实测 数据接近, 但环流的中心位置偏高. NR 模型计算得到的横向流速在垂线方向上 的梯度与实测结果有较大差异, 环流中心出现多个小浴, 与实测流场不符.

(iii) 对于复式断面, SY 模型能够模拟出滩地上多个环流, 而 LY 模型能较好 地模拟出滩槽交界处的二次流动强度, 以及将滩地流动和主槽流动分割开来的 低流速带. 由于弯道情况下离心力的作用, 复式断面弯道流动中一旦形成一个主 要环流, 不论是在滩地上还是主槽中, 其环流方向都是顺时针的. 弯道复式断面 计算结果与顺直渠道量测结果的差别主要表现在滩面和滩槽交界处附近, 该处 计算得出的弯道水流中的紊动强度大大超过顺直渠道的量测结果. 对比矩形断 面的情况, 弯道计算及量测的紊动强度结果与顺直渠道的情况差别并没有如此 明显. 造成这种差别的原因尚有待于进一步研究. 


\section{参考文献}

1 Prinos P, Townsend R, Tavoularies S. Structure of turbulence in compound channel flow. J Hydr Engrg, ASCE, 1985, 111(9):1246 1261

2 Arnold U, Hottges J, Rouve G. Turbulence and mixing mechanisms in compound open channel flow. Proc. 23rd IAHR Congr, Ottawa, A133 A140

3 Tominaga A, Nezu I, Ezaki K, et al. Three-dimensional turbulent structure in straight open channel flows. J of Hyd Res, 1989, 27(1): 149 173

4 Tominaga A, Nezu I. Turbulent structure in compound open-channel flows. J Hydr Engrg, ASCE, 1991, 117(1): 21 41

5 Shiono K, Knight D W. Turbulent open-channel flows with variable depth across the channel. J Fluid Mech, 1991, 222: 617 646

6 Shiono K, Muto Y. Complex flow mechanisms in compound meandering channels with overbank flow. J Fluid Mech, 1998, 376: 221 261

7 Shiono K, Scott C F. Predictions of solute transport in a compound channel using turbulence models. J Hyd Res, 2003, 41(3): 247 258

8 Jia Y F, Wang S S Y. Numerical model of secondary motions of turbulent flows in compound channels. International Water Resources Engineering Conference - Proceedings, 1998, 2: 1038 1043

9 Demuren A O, Rodi W. Calculation of turbulence-driven secondary motion in non-circular ducts. J Fluid Mech, 1984, 140: 189 222

10 Launder B E, Ying W M. Prediction of flow and heat transfer in ducts of square cross-section. Proceedings, Institution of Mechanical Engineers, 1973, 187: 455 461

11 Naot D, Rodi W. Calculation of secondary currents in channel flow. J Hydr Div, ASCE, 1982, 108(8): 948 968

12 Speziale C G. On nonlinear $k-l$ and $k-\varepsilon$ models of turbulence. J Fluid Mech, 1987, 178: 459 475

13 Launder B E, Spalding D B. The numerical computation of turbulent flow. Comp Meth Appl Mech Engng, 1974, 3: 269 289 